\title{
Ambulante Schmerztherapie mit Mängeln
}

\section{Ziel der vorliegenden Studie war es, die Prävalenz von Schmerzen und Analgetika-Anwendung bei ambulanten Krebspatienten zu untersuchen.}

O bwohl Schmerzen ein häufiges und belastendes Symptom bei Krebspatienten sind, ist das Schmerzmanagement oft mangelhaft. In einer großen, vor 20 Jahren durchgeführten Studie der Eastern Cooperative Oncology Group (ECOG) erhielten $42 \%$ der untersuchten Patienten keine adäquate Schmerztherapie. Auch heute noch ist das Schmerzmanagement bei Krebspatienten häufig unzureichend, wie die vorliegende Studie mit 3.123 ambulanten Patienten mit invasiven Tumoren von Brust, Prostata, Kolon, Rektum oder Lunge aus $38 \mathrm{Be}$ handlungszentren in den USA zeigt.

Bei der ersten Untersuchung und vier oder fünf Wochen später hatten die $\mathrm{Pa}$ - tienten Fragebögen zu Schmerzen, funktionellen Beeinträchtigungen und anderen Symptomen ausgefüllt. Um zu untersuchen, ob die Therapie adäquat war, erfolgte die Berechnung des Schmerzmanagement-Index, eines validierten Tools zur Beurteilung der Schmerztherapie.

Bei 3.023 der Patienten bestand ein Risiko für Schmerzen, 2026 (67\%) von ihnen gaben bei der ersten Untersuchung an, dass sie Schmerzen hätten und Analgetika benötigten. $33 \%$ von ihnen berichteten, keine adäquaten Analgetika verordnet zu bekommen. Beispielsweise bekamen $41 \%$ der Patienten mit mäßigen bis starken Schmerzen keine Opioide, $20 \%$ mit starken Schmerzen erhielten überhaupt kein Analgetikum.

Auch bei der zweiten Untersuchung beklagten $34 \%$ eine inadäquate Schmerztherapie beklagten. In weiteren Analysen konnten einige Prädiktoren für eine bessere schmerztherapeutische Versorgung identifiziert werden: Beispielsweise hatten nichthispanische weiße Patienten nur ein etwa halb so großes Risiko für eine inadäquate Therapie wie andere Patienten (Odds Ratio: 0,51; $p=0,002$ ). Signifikante Prädiktoren für eine bessere Versorgung waren auch ein schlechter Performancestatus, die Behandlung in einem Zentrum, in dem vorwiegend nichthispanische weiße Patienten versorgt wurden und das Vorliegen einer behandelten, fortgeschrittenen Erkrankung.

Fazit: Die meisten ambulanten Patienten mit soliden Tumoren sind mit Problemen hinsichtlich Schmerzen und Analgetikaanwendung konfrontiert. Ein Risiko einer Unterversorgung bestand in dieser Studie aus den USA vor allem für ethnische Minderheiten, z.B. Patienten hispanischer Abstammung. Judith Neumaier

Fisch MJ et al. Prospective, observational study of pain and analgesic prescribing in medical oncology outpatients with breast, colorectal, lung, or prostate cancer. J Clin Oncol. 2012;30(16): 1980-8.

\section{Wichtig: Übelkeit und Erbrechen vorbeugen}

\section{Bei hochemetogenen Chemotherapien sollte man der drohenden Übelkeit vorbeugen. Zum dafür breit angewendeten Aprepitant gibt es jetzt eine Weiterentwicklung. Wie sicher und wirksam ist Fosaprepitant?}

$\mathrm{V}_{\mathrm{i}}$ iele Krebspatienten fürchten sich vor Übelkeit und Erbrechen als sehr unangenehmen Nebenwirkungen einer Chemotherapie. Vorbeugend empfehlen derzeit gültige Richtlinien bei Patienten, die eine hochemetogene (zum Beispiel platinbasierte) Chemotherapie erhalten, eine Dreierkombination aus einem 5-HT3-Rezeptorantagonisten, einem Kortikosteroid sowie dem NeurokininTyp-1-Rezeptorantagonisten Aprepitant.

Fosaprepitant, ein wasserlösliches phosphoryliertes Prodrug von Aprepitant, wird, intravenös gegeben, schnell in Aprepitant verwandelt. Eine jetzt vorliegende Studie aus Japan untersucht die Wirksamkeit und Sicherheit einer einmaligen Dosis von Fosaprepitant in Kombination mit intravenös verabreichtem Granisetron und Dexamethason. Allerdings wurde in der Vergleichsgrup- pe Placebo statt Aprepitant verwendet, weil letzteres zu Beginn der Studie in Japan noch nicht zugelassen war.

Aufgenommen wurden Patienten, die eine Cisplatin-basierte Chemotherapie erhielten $\left(\geq 70 \mathrm{mg} / \mathrm{m}^{2}\right)$. Von den insgesamt 347 Patienten hatten $21 \%$ nach Cisplatin bereits unter Erbrechen gelitten. In der Verumgruppe erhielten die Patienten 150 mg Fosaprepitant i.v. an Tag 1 in Kombination mit $40 \mu \mathrm{g} / \mathrm{kg}$ Granisetron an Tag 1 sowie Dexamethason i.v. an den Tagen 1-3. Im Kontrollarm wurde Fosaprepitant durch Placebo ersetzt. Primärer Endpunkt war der Anteil der Patienten, die vollständig ansprachen, also von Stunde 0-120 eines Zyklus keine Emesis erlebten und keine Rescuetherapie benötigten.

Die Zahl der Patienten mit vollständigem Ansprechen war in der Verumgrup- pe signifikant höher als in der Kontrollgruppe (64 vs. $47 \%$; $p=0,0015$ ). Das Fosaprepitant-Regime war sowohl in der akuten Phase (0-24 h nach Chemotherapiebeginn) effektiver als das Kontrollregime (94 vs. $81 \%$ ) als auch in der verspäteten Phase (nach $24-120$ h; 65 vs. $49 \% ; \mathrm{p}=0,0025)$.

Das Toxizitätsprofil der Kombination war mild. Moderate Nebenwirkungen traten in der Fosaprepitant-Gruppe häufiger auf ( 3,4 vs. $1,8 \%$; $p=0,3280)$. Hämatologische Toxizitäten (febrile Neutropenie, Anämie und Thrombozytopenie) waren in beiden Gruppen vergleichbar.

Fazit: Für Patienten, die eine hochemetogene Krebstherapie erhalten, ist Fosaprepitant plus Dexamethason und Granisetron eine gut tolerierte und effektive Option.

Christina Berndt

Saito $\mathrm{H}$ et al. Efficacy and safety of single-dose fosaprepitant in the prevention of chemotherapy-induced nausea and vomiting in patients receiving high-dose cisplatin: A multicentre, randomised, double-blind, placebo-controlled phase 3 trial. Ann Oncol. 2012 Oct 31. [Epub ahead of print] 